\title{
Primary Absence of Type II Endoleak is A Positive Prognostic Factor against the Risk of Late Conversion of EVAR for AAA
}

\author{
Bonardelli $\mathbf{S}^{1}$, Zanotti $\mathbf{C}^{1^{*}}$, Cervi $\mathrm{E}^{1}$, De Lucia $\mathbf{M}^{1}$, Nodari $\mathrm{F}^{1}$, Guadrini $\mathbf{C}^{1}$, Cuomo $\mathbf{R}^{2}$ and Battaglia $\mathbf{G}^{2}$ \\ ${ }^{1}$ Institute of Vascular Surgery, University of Brescia - Italy \\ ${ }^{2}$ Institute of Radiology, University of Brescia - Italy
}

"Corresponding author: Camilla Zanotti, Spedali Civili Hospital, Vascular Surgery- Department of General Surgery III, Piazzale Spedali Civill, Brescia, Brescia 25100, Italy, Tel: +39 3398001782; E-mail: zanotti.camilla@gmail.com

Received date: Sep 6, 2014, Accepted date: Nov 26, 2014, Publication date: Dec 5, 2014

Copyright: $\odot 2014$ Bonardelli S et al. This is an open-access article distributed under the terms of the Creative Commons Attribution License, which permits unrestricted use, distribution, and reproduction in any medium, provided the original author and source are credited.

\begin{abstract}
Introduction: The aim of this study is to analyze 12 late conversion to open surgery after Endovascular Repair of Abdominal Aortic Aneurysms (EVAR) while comparing the follow up of these cases to that of the definitely successful procedures (absence of surgical conversion, type I or III endoleaks, or presence of type II endoleaks without any aneurysmal sac enlargement) .

Methods: From a series of over 300 EVAR procedures performed at our department we have selected 215 cases with a follow up $\geq 6$ month and primary technical success (successful deployment of the devices and discharge of patients without neither type I nor III endoleaks). Based on the final data recorded at the end of the follow up (mean+ IQR: 38.16 months + 41), these cases were divided into three groups: group 1, with 12 cases $(5.6 \%)$ which needed surgical conversion in a later stage ( 5 to 55 months from EVAR); group 2, with 39 cases $(18.1 \%)$ with type II endoleaks without aneurysmal sac enlargement; group 3 , with 164 cases $(76.5 \%)$ without endoleaks. The groups were compared in relation to the following parameters: a) personal data and common atherogenic risk factor, b) diameter of the aneurysm, c) kind of the proximal fixation of the endograft (suprarenal or infrarenal), d) presence of endoleaks at the first postoperative check. We have compared the data from the three groups and we have analyzed them with chi-square test $\left(\mathrm{X}^{2}\right)$.
\end{abstract}

Results: Personal data and common atherogenic risk factor have proved no significant difference among the three groups. The incidence of the other three parameters of group 1 was compared with the incidence of these in groups 2 and 3: the mean pre-operative diameter of the aneurysm results $51 \mathrm{~mm}$ in group $1,54 \mathrm{~mm}$ in group 2 and $55 \mathrm{~mm}$ in group 3 (not significant); suprarenal fixation of the prosthesis accounts for $50 \%$ in group 1, 51\% in group 2 and $60 \%$ in group 3 (not significant); presence of type II endoleak at the first post-operative check was $41.6 \%$ in group $1,56.4 \%$ in group 2 (not significant) and $9.7 \%$ in group 3 ( $p<0.001$, compared to groups 1 and 2 ).

Conclusion: In the EVAR procedures with primary technical success, the absence of type II endoleak at the first post-operative check represents a favorable prognostic factor against the risk of late conversion to open repair. Personal data, common atherogenic risk factor, diameter of the aneurysm and fixing type of the prosthesis don't seem to influence the onset of this complication.

Keywords: Endoleak; EVAR; AAA; Late conversion

\section{Introduction}

Endovascular Abdominal Aortic Repair (EVAR) has established over time as an effective and safe method to treat abdominal aortic aneurysms. This technique ensures a reduced perioperative morbidity and mortality compared to the traditional surgical technique, especially in elderly patients with significant comorbidities $[1,2]$. Patients who have undergone endovascular treatment need lifelong surveillance, in order to prevent and treat potential complications, including endoleak, increase in aneurysm size, graft migration, structural graft failure and limb stenosis or occlusion. The presence of these complications, in a small percentage of cases, can results in a late conversion to open repair [3]. The aim of this study is to analyze 12 EVAR with late conversion in comparison with 203 cases with longterm good results.

\section{Methods}

Our EVAR database compiled in a prospective, real time manner was reviewed. From a series of over 300 consecutively performed EVAR procedures, 215 cases with a follow up $\geq 6$ months were considered. Regarding the indication for EVAR we closely followed the international guidelines, selecting patients with asymptomatic abdominal aortic aneurysm greater than $5 \mathrm{~cm}$ in an orthogonal projection or an aneurysm diameter of $4-5 \mathrm{~cm}$, which has increased in size by $0.5 \mathrm{~cm}$ in last six months or in presence of blister/bubbles. The patient should have a proximal landing zone (distal to the lower renal artery) of at least $1.5 \mathrm{~cm}$ without significant calcification or thrombus, and similarly a distal landing zone of at least $2.5 \mathrm{~cm}$. The proximal neck angulation should be less than 45 degrees, and the patient should have access vessels of at least 6-8 $\mathrm{mm}$ luminal diameter (depending on the manufacturer's requirements) and without extreme tortuosity. The lower diameter of treated aneurysms was $42 \mathrm{~mm}$, the largest $75 \mathrm{~mm}$. In the 215 cases considered, we used the following devices: Anaconda ( 3 
Page 2 of 4

cases), Aneurx (19 cases), Aorfix (4 cases), Endologix (2 cases), Medtronic Endurant (32 cases), Gore Excluder (65 cases), Medtronic Talent (89 cases); Zenith Cook (1 case); between these, the device used in the converted cases was AneuRx ( 3 cases), Excluder ( 1 case) or Talent (8 cases).

Usually, we recommend to all patients undergoing EVAR antiplatelet therapy (with acetylsalicylic acid or ticlopidine or clopidogrel) if it is not already in use oral anticoagulant therapy. All selected cases had obtained primary technical success (successful deployment of the devices in absence of surgical conversion, type I or III endoleaks). The follow up protocol included clinical and instrumental examinations at 3,6,12 months and yearly thereafter. All patients underwent follow-up by CT angiography at first. If after 2-3 consecutive CT checks there was no evidence of endoleaks and there was shrinkage of the excluded aneurysm sac, we performed the following control by Duplex Ultrasound. We used magnetic resonance imaging in cases of allergy to iodinated contrast.

Based on the last data collected at the end of the follow up, the 215 cases were divided into three groups: group 1, with 12 cases $(5.6 \%)$ with type I (4 cases), II(7 cases) or III(1 case) endoleaks and aneurysmal sac enlargement, and therefore ended with open surgical conversion; group 2, including 39 cases (18.1\%) with type II endoleaks without aneurysmal sac enlargement; group 3, including 164 cases (76.5\%) without endoleaks. Each group was evaluated in relation to personal data and common atherogenic risk factor, diameter of the aneurysm, fixation of the prosthesis (suprarenal or infrarenal), and presence of endoleaks at the first postoperative control. At the end the characteristics of the 12 converted cases were compared to that of the successful procedures of Group 2 and 3.

\section{Results}

In group 1 there were 4 cases of type I endoleak not treatable with endovascular skills and 7 cases of type II endoleak with sac enlargement, 1 case of type III endoleak subsequent to a persistent type II endoleak lasting more than 2 years after EVAR. Out of seven cases of type II endoleaks, 2 cases weren't suitable for angiographic treatment, because of an unfavorable anatomy of the ileo-lumbar circle, 4 had been subjected to unsuccessful angiographic treatment (lumbar arteries embolization with coils); 1 patient had undergone inferior mesenteric artery laparoscopic ligation. The kind of device used in this group of patients was AneuRx (3 cases), Excluder (1 case) or Talent ( 8 cases). The average time between EVAR and conversion to open repair was 55.6 months (range 5 - 120 months) (Table 1). The comparison of the groups in regards to personal data of the patients, common atherogenic risk factors, mean diameters of the aneurysms and kind of proximal fixation of the endoprosthesis has not revealed significant differences (Table 2): male gender was $100 \%$ in group 1, $93.8 \%$ in group 2 and $94.1 \%$ in group 3 . The average age of the patients was 74.6 years in group 1, 73.1 years in group 2, 75.2 years in group 3. Smoking History was present in $41.6 \%$ of patients in group $1,44.8 \%$ in group 2 and $41.3 \%$ in group 3. Hypertension affected $75 \%$ of patients in group 1, 73.4\% in group 2 and $72.4 \%$ in group 3 . Renal Insufficiency was present in $16.7 \%$ of patients in group 1,16.3\% in group 2 and $10 \%$ in group 3. The mean pre-operative diameter of the aneurysm resulted $51 \mathrm{~mm}$ in group $1,54 \mathrm{~mm}$ in group 2 and $55 \mathrm{~mm}$ in group 3 . Suprarenal fixation of the prosthesis accounted for $50 \%$ in group $1,51 \%$ in group 2 and $60 \%$ in group 3 . The only significant difference between the analyzed groups consists in the low rate of type II endoleak at the first post-operative check in Group 3 (9.7\%), compared both with $41.6 \%$ in Group 1 and 56.4\% in Group 2 ( $\mathrm{p}>0.001)$.

\begin{tabular}{|l|l|}
\hline Features of 12 Converted Cases (group 1) \\
\hline Endoleak & Type i -4 \\
\cline { 2 - 3 } & Type ii -7 \\
\cline { 2 - 3 } & Type iii -1 (previous type ii endoleak) \\
\hline Device & Aneurx -3 \\
\cline { 2 - 3 } & Talent -8 \\
\cline { 2 - 2 } & Excluder -1 \\
\hline Average time (month) between evar and conversion & 55.6 \\
\hline Treatment of type ii endoleak & Lumbar arteries embolization -5 \\
\cline { 2 - 2 } & Failed attempts to embolization -2 \\
\cline { 2 - 2 } & Ami laparoscopic ligation -1 \\
\hline
\end{tabular}

Table 1: Features of the Converted Cases

\begin{tabular}{|l|l|l|l|}
\hline & Group 1 & Group 2 & Group 3 \\
\hline $\mathrm{N}^{\circ}$ Patient & 12 & 39 & 164 \\
\hline a) Pre-Operative Diameter & 51 & 54 & 55 \\
\hline b) Device Fixation & & \\
\hline
\end{tabular}




\begin{tabular}{|c|c|c|c|}
\hline Suprarenal & 6 & 20 & 99 \\
\hline Infrarenal & 6 & 19 & 65 \\
\hline \multicolumn{4}{|c|}{ c) Type li Endoleak (1st post-operative control) } \\
\hline No & 7 & 17 & 148 \\
\hline Yes & 5 & 22 & 16 \\
\hline
\end{tabular}

Table 2: Comparison between the 3 groups

\section{Discussion}

Starting from the first interventions more than 20 years ago [4], technological advances have allowed EVAR to become the most common method to treat infrarenal abdominal aortic aneurysm with good short term and acceptable long term outcomes, as reported in literature [1]. Despite technological advances, a fair percentage of patients need further treatment following EVAR and this is the reason why lifelong radiographic surveillance is mandatory after this kind of procedure [5]. In the randomized EVAR-1 trial, the rate of late conversion (beyond the initial 30 postoperative days) to open repair was $2.6 \%$, with a mean delay of 3.3 years after first repair [1]. Late conversion has been reported in literature as the follow up to $9 \%$ of EVAR [6]: our conversion rate (5.58\%) falls within that range.Data emerging from our series suggest that age, gender, common atherogenic risk factors of the patients, as well as diameter of the aneurysm and type of endograft proximal fixation, do not significantly affect the post-operative outcome. The presence of type II endoleak at the first post-operative control seems to be related with late adverse outcome after EVAR (a statistically significant difference between group 3 and group 1 and 2 is observed). It is rather common knowledge that clinical impact of type II endoleak after EVAR is not well established and remains highly controversial [7-10]. First of all, the incidence of type II endoleak oscillates greatly from $6 \%$ to up to $30 \%$ in large series of EVAR patients $[11,12]$. Secondly, published reports demonstrated that spontaneous resolution of endoleaks can sometimes occur, even if this is not associated with constant rates of occurence (5-33\%) [13]. Furtherly, most of the authors assert that type II endoleak is a minor complication not necessarily related to an increase in aneurysms size [14-16]. Actually, EUROSTAR series had reported a significantly higher rate of re-intervention required by patients with type II endoleaks, but did not find a direct correlation between this type of endoleak and conversion to open repair or rupture. Jones at al demonstrated the negative impact of type II endoleaks in increasing risk of aneurysm rupture, sac grow, conversion to open repair, compared with patients without endoleaks [17]. In the series reported by Jones et al. 3 out of 33 patients with a persistent type 2 endoleak required a conversion to open repair, while aneurysm rupture occurred in four patients with an early type 2 endoleak. Statistical analysis showed them that patients with a persistent type 2 endoleak had a significantly higher rate of conversion to open repair compared with those without an early endoleak (RR, 5.3; $95 \%$ CI, 2.0 to 13.5 ; P 0.001 ) and patients with a persistent type 2 endoleak had a higher rate of rupture compared to those without early endoleak (RR, 3.9; 95\% CI, 1.7 to 8.8; P 0.03). Our data does not confirm Jones' conclusions, because we found a significant rate of type II endoleak also in group 2, which doesn't show any sac enlargement: $18.1 \%$ to $56.1 \%$ at the first postoperative check, but still significant at the end of the follow up. On the other hand, the significant lower rate of primary type II endoleak in group 3 (9.6\%) leads us to believe that the absence of this kind of leak after EVAR is an effective protective factor from the risk of late conversion. Recently, Nolz et al. stated that - in the long term - follow up persistent type II endoleak leads to significant aneurysm sac enlargement in comparison with aneurysm with transient endoleak or its absence. Close surveillance seems to be advisable in aneurysms with persistent type II endoleak only [18]. Therefore, just after the first check, it would seem sensible to put the majority of efforts into performing a strict follow up in cases with endoleak II only, whilst it would be reasonable to reduce the frequency of the subsequent checks in patients who show the protective factor, in the form of the absence of type II endoleak at the first postoperative check.

\section{Conclusion}

In the EVAR procedures with primary technical success, the absence of type II endoleak at first post-operative check represents a favorable prognostic factor against the risk of late conversion to open repair and it allows to reduce the rate of subsequent radiological checks, while personal data, common atherogenic risk factor, diameter of the aneurysm and fixing type of the prosthesis don't seem to influence the onset of this complication.

\section{References}

1. EVAR trial participants (2005) Endovascular aneurysm repair versus open repair in patients with abdominal aortic aneurysm (EVAR trial 1): randomised controlled trial. Lancet 365: 2179-2186.

2. Prinssen M, Verhoeven EL, Buth J, Cuypers PW, van Sambeek MR, et al. (2004) A randomized trial comparing conventional and endovascular repair of abdominal aortic aneurysms. N Engl J Med 351: 1607-1618.

3. Moulakakis KG, Dalainas I, Mylonas S, Giannakopoulos TG, Avgerinos ED, et al. (2010) Conversion to open repair after endografting for abdominal aortic aneurysm: a review of causes, incidence, results, and surgical techniques of reconstruction. J Endovasc Ther 17: 694-702.

4. Parodi JC, Criado FJ, Barone HD, Schönholz C, Queral LA (1994) Endoluminal aortic aneurysm repair using a balloon-expandable stentgraft device: a progress report. Ann Vasc Surg 8: 523-529.

5. Forbes TL1, Harrington DM, Harris JR, DeRose G (2012) Late conversion of endovascular to open repair of abdominal aortic aneurysms. Can J Surg 55: 254-258.

6. Kelso RL, Lyden SP, Butler B, Greenberg RK, Eagleton MJ, et al. (2009) Late conversion of aortic stent grafts. J Vasc Surg 49: 589-595.

7. Zarins CK, White RA, Hodgson KJ, Schwarten D, Fogarty TJ (2000) Endoleak as a predictor of outcome after endovascular aneurysm repair: AneuRx multicenter clinical trial. J Vasc Surg 32: 90-107.

8. Ohki T, Veith FJ, Shaw P, Lipsitz E, Suggs WD, et al. (2001) Increasing incidence of midterm and long-term complications after endovascular graft repair of abdominal aortic aneurysms: a note of caution based on a 9-year experience. Ann Surg 234: 323-334. 
Citation: Bonardelli S, Zanotti C, Cervi E, De Lucia M, Nodari F, et al. (2014) Primary Absence of Type II Endoleak is A Positive Prognostic Factor against the Risk of Late Conversion of EVAR for AAA. Angiol Open Access 2: 139. doi:10.4172/2329-9495.1000139

Page 4 of 4

9. Maldonado TS, Gagne PJ (2003) Controversies in the management of type II "branch" endoleaks following endovascular abdominal aortic aneurysm repair. Vasc Endovascular Surg 37: 1-12.

10. Buth J, Harris PL, van Marrewijk C, Fransen G (2003) The significance and management of different types of endoleaks. Semin Vasc Surg 16: 95-102.

11. Laheij RJ, Buth J, Harris PL, et al. (2000) Need for secondary interventions after endovascular repair of abdominal aortic aneurysms. Intermediate-term follow-up results of a European collaborative registry (Eurostar). Br J Surg 87:1666-1673

12. Gelfand DV, White GH, Wilson SE (2006) Clinical significance of type II endoleak after endovascular repair of abdominal aortic aneurysm. Ann Vasc Surg 20: 69-74.

13. Sheehan MK, Ouriel K, Greenberg R, McCann R, Murphy M, et al. (2006) Are type II endoleaks after endovascular aneurysm repair endograft dependent? J Vasc Surg 43: 657-661.

14. Steingruber IE, Neuhauser B, Seiler R, Greiner A, Chemelli A, et al. (2006) Technical and clinical success of infrarenal endovascular abdominal aortic aneurysm repair: A 10-year single-center experience. Eur J Radiol 59: 384-392.

15. Rayt HS, Sandford RM, Salem M, Bown MJ, London NJ, et al. (2009) Conservative management of type 2 endoleaks is not associated with increased risk of aneurysm rupture. Eur J Vasc Endovasc Surg 38: 718-723.

16. Veith FJ, Baum RA, Ohki T, Amor M, Adiseshiah M, et al. (2002) Nature and significance of endoleaks and endotension: summary of opinions expressed at an international conference. J Vasc Surg 35: 1029-1035.

17. Jones JE, Atkins MD, Brewster DC, Chung TK, Kwolek CJ, et al. (2007) Persistent type 2 endoleak after endovascular repair of abdominal aortic aneurysm is associated with adverse late outcomes. J Vasc Surg 46: 1-8.

18. Nolz R, Teufelsbauer H, Asenbaum U, Beitzke D, Funovics M, et al. (2012) Type II endoleaks after endovascular repair of abdominal aortic aneurysms: fate of the aneurysm sac and neck changes during long-term follow-up. J Endovasc Ther 19: 193-199. 